\title{
Caso Garittea, del campo al campus: Creación del diseño de la identidad visual de una organización a través del trabajo colaborativo entre comunidades campesinas y la academia.
}

\author{
Rosales, Elingth Simonéa ; Mora, Claudia Lucía ${ }^{\mathrm{b}}$; Mora, Florencia ${ }^{\mathrm{c}}$; Morales, Rocío ${ }^{\mathrm{d}}$ \& Bermúdez, \\ Diego Giovanni ${ }^{\mathrm{e}}$ \\ ${ }^{a}$ Universidad Pontificia Javeriana Cali, Colombia. elingthsimone@gmail.com \\ ${ }^{\mathrm{b}}$ Universidad Pontificia Javeriana Cali, Colombia. cmora@javerianacali.edu.co \\ ${ }^{\mathrm{c}}$ Universidad Pontificia Javeriana Cali, Colombia. fmoraa@javerianacali.edu.co \\ d Universidad Pontificia Javeriana Cali, Colombia.rmorales@javerianacali.edu.co \\ ${ }^{\text {e}}$ Universidad Pontificia Javeriana Cali, Colombia. dgbermudez@javerianacali.edu.co
}

\begin{abstract}
Resumen
Se presenta la experiencia de articulación entre academia, ONG y asociaciones campesinas, alrededor del desarrollo de capacidades para la comercialización de productos de café, en el marco del diseño colaborativo y participativo, que permite avanzar en la construcción de canales de economía solidaria y comercio justo. Garittea es una empresa social que disminuye la intermediación comercial, defiende los ingresos de los pequeños caficultores y genera procesos de empoderamiento en las comunidades campesinas; es una marca creada para el fortalecimiento de las relaciones campo-ciudad, el desarrollo sostenible, el impulso del trabajo interdisciplinario y colaborativo, elementos esenciales en la creación de la identidad y el diseño de comunicación visual de una organización. Villafañe (2008) afirma que la identidad corporativa es el "ser" de la empresa, su esencia, concepto que se toma como referencia y pone de manifiesto la importancia del trabajo colectivo en la creación de la identidad en las organizaciones.
\end{abstract}

Este estudio deja ver los modos de participación e intervención de los distintos actores y áreas del conocimiento en los procesos de diseño y gestión de una organización, generando el posicionamiento y la visibilización del carácter de una comunidad productiva. La identidad de Garittea parte de una visión integradora articulada con perspectiva de la triple hélice (Etzkowitz et al. 2000) donde empresas, universidades y otras organizaciones se encuentran para trabajar con un compromiso colaborativo.

Los actores del proceso se definen a través de una triada, compuesta por la empresa, (socios de Garittea): la Asociación de Pequeños Caficultores de La Marina, la Asociación de Caficultores Orgánicos de Colombia y el Instituto Mayor Campesino, quienes han aportado su know-how y la experiencia de sus procesos de creación de nuevos productos; un equipo de $I+D+i$ (profesores y gestores), quienes han incorporado sus metodologías y herramientas a través de la interdisciplinariedad en saberes como el mercadeo, la ingeniería, la economía, la psicología y el diseño, aplicando entre otros recursos, modelos 
Caso Garittea, del campo al campus: Creación del diseño de la identidad visual de una organización a través del trabajo colaborativo entre cominidades campesinas y la academia,

sistémicos de formulación por objetivos para apoyar los procesos creativos y la gestión del diseño; los diseñadores (estudiantes), quienes han trabajado en la creación de la identidad de Garittea a través de un equipo integral, dando como resultado la creación de la identidad corporativa e identidad visual de Garittea.

Palabras clave: Identidad visual corporativa, entornos colaborativos, diseño participativo campo - ciudad, sistémica.

\begin{abstract}
This paper presents the joint work experience between academics, ONGs, and farmer's associations, about the development of capabilities for the marketing of coffee products, in the framework of colaborative and participative design, that allows the progress in the construction of solidary economic chanels and fair trade. Garittea is a social entrepreneurship that lowers commercial intermediation, defends the income of small coffee producers, and creates empowerment processes in the rural comunities; Garittea is a brand created for strengthtening rural-urban relations, sustainable development, the promotion of interdisciplinary and colaborative work, esential elements in the creation of business identity and the design of an organization visual comunication. Villafañe (2008) states that corporative identity is the "being" of a company, its essence, a concept that is taken as a reference and highlights the importance of collective work in the creation of identity of organizations.
\end{abstract}

This study reveals the several ways of participation and involvement of different actors and areas of knowledge in the processes of design and management of an organization, that creates positioning and visibility of the character of a productive community. The identity of Garittea departs from an integrated vision, articulated with triple helix perspective (Etzkowitz et al. 2000) where companies, universities, and other organizations are willing to work jointly with a colaborative commitment.

The actors in the process are defined by a triad, the firm (Garittea shareholders): the Association of Small Coffee Growers of La Marina, the Association of Colombian Organic Coffee Growers and the Instituto Mayor Campesino, who have contributed with their knowhow and experience in the processes of creating new products; a team of $I+D+i$ (teachers and administrators), who have incorporated their methodologies and tools through interdisciplinary knowledge in areas such as marketing, engineering, economics, psychology, and design, using among other resources, systemic models of formulation by objectives to support the creative process and the design management; and, finally, the designers (the students), who have worked on creating Garittea's identity through an integrated team, resulting in the creation of corporate identity and visual identity of Garittea.

Keywords: corporate visual identity, colaborative environments, rural-urban participative design, sistemic. 


\section{La experiencia. Actores y tensiones}

"Profesor, no nos interesan sus planteamientos de un negocio que se fundamenta en la lógica del mercado, para nosotros la vida se mueve en la construcción de posibilidades de encuentro y cooperación...” Líder campesino (2014)

Ubicar el contexto de la experiencia creativa asociada al proyecto del café, implica re-leer un proceso construido conjuntamente, entre académicos, funcionarios de una ONG y líderes campesinos. Volver una y otra vez sobre la experiencia de encuentro que hemos construido, ha permitido constatar las distintas tensiones sobre las que se ha establecido la construcción de un proyecto denominado Garittea, del campo al campus. Tensiones que bien vale la pena explicitar cuando abordamos el encuentro desde dos lógicas: el valor de los emprendimientos medido por las ganancias, el empleo que genera, la acumulación y la aplicación de modelos previamente establecidos, que marcan resultados "éxitosos" en el mercado; y la concepción de los proyectos productivos generadores de encuentros, posibilidades, recreación de la cultura, cuidado de la naturaleza y compromiso con la vida. En palabras de Albarracin (2009, p. 19) “quizás ese es el punto más conflictivo de una aproximación de este tipo de iniciativas, ya que su correcta lectura precisa el deslizamiento de un terreno claramente definido por la materialidad del dinero, las mercancías, la acumulación, a otro, fangoso e inhóspito y acechado por el fantasma de un concepto tan vago como el del capital cultural"

Pareciera que esta dinámica social que se establece entre los saberes hegemónicos y los saberes populares, no termina de transformarse; García Canclini (1990, p. 221) lo ha señalado desde hace más de dos décadas, cuando menciona: "el avance económico moderno no implica eliminar las fuerzas productivas que no sirven directamente a su expansión si esas fuerzas cohesionan a un sector numeroso, aún satisfacen necesidades sectoriales o las de una reproducción equilibrada del sistema. A la inversa y complementariamente, la reproducción de las tradiciones no exige cerrarse a la modernización”.

De igual manera, García-Canclini hace referencia a una serie de estudios en América Latina (México y Ecuador), que ponen en evidencia que las dinámicas económicas, políticas y sociales perpetúan la discriminación y desigualdad de oportunidades para los grupos étnicos y campesinos para acceder a bienes económicos, a educación media y superior pertinente y de calidad, y a nuevas tecnologías; entonces, ¿cuáles son los caminos que deben recorrer comunidades indígenas, afro, campesinas para mantener las tradiciones, participar de la vida urbana y de las reglas comerciales modernas?

En esta perspectiva, la integración de algunos grupos tradicionales con la modernidad ha implicado, como lo menciona García-Canclini (1990, Págs 222-223), una consolidación del sujeto político y social. Es evidente que existen múltiples maneras de construcción de vínculos entre lo tradicional y lo moderno, lo popular y lo culto, lo local y lo extranjero; así como las clases hegemónicas ajustan sus saberes, al mismo tiempo, "las clases populares adoptan sus saberes y hábitos tradicionales". Sin mayor conflicto, entonces plantea García Canclini, la importancia de los cruces culturales y la reestructuración de los vínculos, especialmente, en las nuevas generaciones, más expuestas al mundo globalizado. Allí, el diseño tiene la oportunidad de jugar un papel de gran importancia en este tipo de procesos.

Si bien los planteamientos de García Canclini se realizan en función del arte versus las artesanías, para el caso que nos ocupa nos enfrenta a reflexiones similares aunque más adaptadas a las lógicas y saberes de los habitantes de las urbes versus los habitantes de la zona rural, específicamente, de los campesinos con sus cosmovisiones, luchas y resistencias. Precisamente estos saberes que provienen de la memoria común y cotidiana, y brotan de la dimensión social sensible, se configuran como proyectos estéticos pues proponen modos de hacer, maneras de habitar o circular, formas de interacción que invitan a la elaboración conjunta de sentido. 
Caso Garittea, del campo al campus: Creación del diseño de la identidad visual de una organización a través del trabajo colaborativo entre cominidades campesinas y la academia,

Ya Michel De Certeau (2006), lo señala: "la práctica cotidiana es relativa a las relaciones de fuerza que estructuran el campo social como el campo del conocimiento". De igual manera, De Certeau propone abordar la cultura, en función de objetivos y relaciones, apropiando informaciones, selecccionado y ordenando, bajo la consideración de tres aspectos: estético, polémico y ético.

En efecto, De Certeau, (2006. p. 263) afirma:

"En sí misma, la cultura no es la información, sino su tratamiento mediante una serie de operaciones en función de objetivos y relaciones sociales. Un primer aspecto de estas operaciones es estético: una práctica cotidiana abre un espacio propio en un orden impuesto, como lo hace la acción poética que pliega a su deseo al uso de la lengua común, en un nuevo uso transformador. Un segundo aspecto es polémico: la práctica cotidiana es relativa a las relaciones de fuerza que estructuran el campo social como el campo del conocimiento. Apropiarse informaciones, ponerlas en serie, editarlas a su gusto, es cobrar poder sobre un conocimiento y dar vuelta, de esa forma, a la puesta de imposición de lo ya hecho y ya organizado. Equivale a trazar, con estas operaciones apenas decibles, apenas nombrables, su propio camino en la resistencia del sistema social. Un último aspecto es el ético: la práctica cotidiana restaura con paciencia y tenacidad un espacio de juego, un intervalo de libertad, una resistencia a la imposición (de un modelo, de un sistema o de un orden): poder hacer es tomar distancias, defender la autonomía de algo propio".

En el mismo sentido, el geógrafo Milton Santos, explica cómo la globalización es espacio de flujos diversos: hegemónicos, hegemonizados, rápidos y lentos; los flujos conforman verticalidades y horizontalidades que se entretejen en distintas direcciones. Las horizontalidades se componen de las acciones cotidianas de individuos o instituciones, que responden al ejercicio de existencias solidarias, son lugares que propician relaciones sociales y que fundan lazos solidarios de ciudadanía; las verticalidades son acciones hegemónicas, son elementos perturbadores que intentan traspasar las lógicas de aquellas horizontalidades que se construyen a sí mismas, desde la confianza, desde los afectos, desde la la posibilidad de erigirse como actores sociales colectivos, que se empoderan a partir de sus relaciones. Para Santos (2000), entonces, los cambios vendrán de abajo, desde el territorio, el trabajo y lo cotidiano, a partir de una toma de conciencia de los efectos excluyentes y la retoma de la solidaridad como fundamento de las relaciones sociales. Así, la horizontalidad que se teje desde el espacio-tiempo, desde lo rural y lo urbano, permite el surgimiento de lo político que se fortalece en la dimensión social y sensible de la multiplicidad de actores.

En este contexto, el desarrollo del proyecto Garittea ha estado dinamizado por la interacción de tres actores: el Instituto Mayor Campesino- IMCA-, la Universidad Javeriana Cali y las asociaciones de campesinos- la Asociación de Pequeños Caficultores de La Marina ASOPECAM, la Asociación de Caficultores Orgánicos de Colombia ACOC; todos estos sectores, han intercambiado perspectivas, lo mismo que intereses y afectos, para definir el alcance del proyecto y los modos de su realización, que suponen procesos de construcción y deconstrucción, continuos, como lo hemos mencionado anteriormente.

Ahora bien, esta iniciativa se enmarca en varias plataformas de articulación:

1. La más evidente está referida al trabajo colectivo entre comunidades campesinas, funcionarios de la ONG y académicos. Perspectiva que evidencia el intercambio, interdisciplinar (ingenieros, administradores, diseñadores de la comunicación visual, arquitectos, abogados), y de saberes populares y académicos, "facilitando el aprovechamiento de recursos y promoviendo la interrelación con otras disciplinas actores y saberes"(Gil, J. 2009, p.31).

2. Otra lectura podría ampliarse a las diferentes redes donde cada uno de estos actores participa y genera procesos colectivos, pues "las redes no remiten solamente a una forma de operar, sino 
que suponen nexos conceptuales y espacios de complicidad que trascienden la mera mecánica administrativa" (Gil, J., 2009, pág.31). Situación que nutre el proceso emprendido, como bien lo plantea Yudice (Citado por Javier Gil, (2009) p. 31) "las redes aportan un minucioso trabajo de articulación del cual no son capaces las instituciones modernas. Entra además en espacios a donde no llegan las instituciones...se permitiría que el protagonismo de la acción cultural provenga de la sociedad civil misma". ${ }^{34}$

3. Por su parte la Universidad, identifica en esta alianza la posibilidad de crear un "laboratorio vivo", que impacte directamente el compromiso de docentes y la formación de estudiantes en la perspectiva de su compromiso social, tal como reza su Misión: "la creación y el desarrollo de conocimiento y de cultura en una perspectiva crítica e innovadora, para el logro de una sociedad justa, sostenible, incluyente, democrática, solidaria y respetuosa de la dignidad humana." ${ }^{\text {35 }}$

El derrotero que se ha establecido está en función de integrar dos ejes: la perspectiva campo-ciudad alrededor de un proyecto de economía solidaria y comercio justo en el campus universitario, y explicitar las diferentes formas del trabajo articulado academia, ONG, en este caso el IMCA, y comunidades campesinas. Y además, la perspectiva de la construcción sostenible, en tanto el espacio donde funcionará la tienda de café corresponde a la Casa Alero, casa que obtuvo varios premios, entre ellos el primer lugar en la categoria en Ingeniería y Construcción y Balance Energético, en la competencia mundial del Solar Decathlon Latin American and Caribbean 2015. Esta edificación completa el ciclo del laboratorio vivo que se quiere crear, facilitando mayor apropiación de una propuesta económica de construcción sostenible, éticamente responsable, portadora de solidaridad conjunta y por tanto, promotora de esperanzas.

De esta manera, la Asociación de Pequeños Caficultores de La Marina ASOPECAM, la Asociación de Caficultores Orgánicos de Colombia ACOC, el Instituto Mayor Campesino IMCA y la Universidad Javeriana Cali, vienen trabajando en la construcción de una empresa social que bajo los principios de la economía solidaria, contribuye a eliminar la intermediación comercial, a defender los ingresos de los pequeños productores de café, para potenciar capacidades en las comunidades campesinas, a través de la comercialización directa al consumidor final y la compra de café a un precio justo, impactando el proceso formativo de los estudiantes javerianos, potenciando, además, la construcción de un modelo de consumo ético y sostenible, en la comunidad educativa.

El canal que permitirá que estos objetivos sociales y formativos puedan cumplirse, se materializará en la tienda especializada de café en el campus universitario denominada Garittea del campo al campus, la cual generará un espacio de creación de posibilidades y de gestión de productos propios del mundo campesino y del encuentro híbrido entre las culturas rurales y urbanas.

La comercialización del café, está dada en la creación de posibles y nuevas formas de encuentro entre la ciudad y el mundo rural, entre los jóvenes universitarios y los campesinos, entre los modelos de gestión y la creación, alrededor del fortalecimiento de valores. En palabras de Javier Gil, este espacio de la tienda Garittea puede ser una invitación “ a producir lo nuevo, a generar nuevas relaciones con la economía, la

\footnotetext{
${ }^{34}$ A manera de ejemplo, el Instituto Mayor Campesino junto con otros Centros Sociales orientados por la Compañía de Jesús en Iberoamérica, comparten la misión de acompañar a las personas empobrecidas en su articulación social, política y económica, para que recuperen el control sobre los procesos de desarrollo y mejoren sus condiciones de vida, se han unido y han creado la comunidad de aprendizaje COMPARTE. Con la orientación de la Comunidad COMPARTE y bajo la metodología participativa de lectura estratégica del territorio se ha identificado la producción y comercialización directa de cafés especiales como un proyecto económico-productivo, que de acuerdo con las potencialidades y retos de la región centro del Valle del Cauca, puede generar una remuneración justa e incrementar los ingresos de las familias campesinas de esta zona del Valle.

${ }^{35}$ Consejo Directivo Universitario. Acuerdo N 576. Abril 26 de 2013.
} 
Caso Garittea, del campo al campus: Creación del diseño de la identidad visual de una organización a través del trabajo colaborativo entre cominidades campesinas y la academia,

política, la educación, la vivencia del tiempo y del trabajo, la relación con las comunicaciones; tal vez implica nuevas maneras de estar juntos y de construir comunidades" ( pág. 28)

En su fase inicial, este proyecto favorece la condición social de 61 familias campesinas ubicadas en la zona centro del Valle del Cauca, Colombia, con la visión de integrar en el mediano plazo otras organizaciones campesinas e indígenas para que circulen sus productos de origen a través de este canal de comercialización solidario. Al mismo tiempo, el proyecto genera impacto académico con la oferta de espacios curriculares, como trabajos de grado e investigaciones que continúen desarrollándose alrededor de los ejes articuladores siguientes: consumo responsable, economía solidaria y comercio justo en la perspectiva del fortalecimiento de relaciones culturales campo-ciudad.

\section{Requerimientos de diseño}

El proyecto Garittea del campo al campus, tuvo hacia 2014 su primera intervención por parte de estudiantes de la Carrera de Diseño de Comunicación Visual de la Pontificia Universidad Javeriana Cali, y la necesidad se centró principalmente en la definición de un nombre (naming) y el desarrollo de un logotipo que identificara esta iniciativa de trabajo ya que hasta mediados de 2014 ésta era conocida como el proyecto del café, y no evidenciaba las particularidades y valores de un planteamiento de estas características. Lo anterior se enmarcó en la necesidad de lograr un nivel de identificación del proyecto dentro de la comunidad académica de la Universidad, así como dentro de las comunidades campesinas, las ONG vinculadas a esta iniciativa y el público objetivo al cual se quería llegar en el futuro.

A partir de ello, se inició un proceso de formulación de estrategias de identificación que fuera de la mano con los criterios fundamentales del proyecto con el objetivo de comunicar (poner en común) una propuesta de trabajo colaborativo y participativo con criterios de sostenibilidad, justicia, economía solidaria, autonomía, naturaleza, diálogo de saberes, respeto al medio ambiente, comunidad, entre otros. De esta manera, los diseñadores en formación pusieron en juego sus competencias profesionales, las cuales, se asumieron desde el conocimiento y las capacidades enmarcadas en un saber hacer, un saber analizar, un saber actuar y un saber decidir en contexto.

El ejercicio se llevó a cabo inicialmente en la clase de Diseño de Comunicación Visual, aunque posteriormente participaron otras asignaturas, como Identidad de la Marca en los Empaques. Se partió de la estructuración de un brief que sirviera para conocer y estudiar todos los componentes iniciales necesarios para llevar a cabo el proyecto de diseño, tomando como base la definición de los siguientes puntos a partir de la metodología propuesta por Phillips (2004):
a. Descripción general del proyecto
b. Objetivos del proyecto
c. Campo de intervención
d. Contexto y antecedentes
e. Audiencia objetivo
f. Requerimientos de diseño
g. Alcance de la propuesta
h. Entregables

Antes de abordar la definición del brief, los estudiantes iniciaron sus indagaciones sobre el proyecto del café estudiando las cuatro dimensiones de la identificación institucional: Realidad, Comunicación, Identidad e Imagen (Chaves, 2001). El objetivo se centró en el conocimiento a detalle de las características y componentes básicos que defieran el proyecto, en el cual se pudieran observar las 
dimensiones de este ente social cuya representación manifiesta el discurso de identidad de un sujeto diseñado.

Al aproximarse a la Realidad del proyecto (conjunto de rasgos y condiciones objetivas del ente social), se intentó dar respuesta a la pregunta quién es el ente social. Allí se estudiaron la modalidad organizativa del proyecto del café, su historia, su definición jurídica, sus componentes administrativos, su infraestructura de funcionamiento, entre otras. Los hallazgos permitieron observar un proyecto con una tendencia evolutiva, en el que se configuró un proceso en su dimensión operativa y en sus objetivos de trabajo a futuro.

Posteriormente se analizó la Identidad del proyecto, la cual está determinada como un fenómeno de la conciencia en respuesta a la pregunta de quién quiere ser el ente social. Este aspecto asumido como una forma de autorepresentación, se manifiesta por medio del conjunto de atributos asumidos como propios por el proyecto del café donde se hace evidente la filosofía del proyecto, sus principios de personalidad, sus ideales y sus valores como sujeto social.

A continuación, los estudiantes realizaron un estudio sobre la Comunicación, dimensión semiótica del proyecto (conjunto de mensajes efectivamente emitidos de manera consciente e inconsciente por parte del ente social). Allí, se analizaron los tipos de mensajes que el proyecto del café debe emitir con el fin de dar respuesta a las necesidades de comunicación de un proyecto de estas características (qué se quiere decir fue la pregunta orientadora). A partir de lo anterior, los estudiantes pensaron en los discursos a presentar, los medios a utilizar, así como en las múltiples audiencias que el proyecto debía considerar en la construcción de su identidad corporativa.

Finalmente, se trabajó en la Imagen, última dimensión dentro del esquema propuesto por Chaves (2001) en la construcción del registro público de los atributos identificatorios del proyecto del café. Así, se determinó la lectura y la interpretación que de ella hace la audiencia de modo intencional o espontáneo. Este punto definió lo que los diseñadores desean dejar en la mente del público objetivo del proyecto, aspecto vital y esencia en todo proyecto de identidad visual corporativa.

Teniendo como fundamento principal la información recopilada y construida sobre los cuatro dimensiones anteriores, se dio inicio al trabajo de construcción del brief, en el que fue esencial la participación de todos los actores del proyecto del café (comunidades de caficultores, ONG y academia). Las particularidades de cada una de las partes integrantes de este proyecto, se hicieron evidentes en las reuniones de trabajo del proyecto, expresando la manera en como cada grupo interpretaba las necesidades que debía dar respuesta el ejercicio de diseño. Los campesinos manifestaron su interés para que el proyecto de identidad corporativa acogiera el saber tradicional campesino, sus expresiones cotidianas, la riqueza natural de su entorno y diera cuenta además del proceso de producción del café. Por su parte, el Instituto Mayor Campesino abogó por un discurso de identidad que tuviera fortaleza desde el punto de vista comercial, sin dejar a un lado la representación del trabajo comunitario que caracteriza a esta iniciativa. La Universidad, manifestó su interés para que el proyecto diera cuenta del diálogo de saberes, así como de los múltiples públicos objetivos que se verían involucrados en una iniciativa como ésta.

Los objetivos del proyecto de diseño se enmarcaron en una primera fase, en la articulación de un nombre para esta experiencia de trabajo, bajo los requerimientos anotados anteriormente. Posteriormente, se debía desarrollar un logotipo que representara la diversidad y multiplicidad del proyecto del café, bajo criterios de recordación, claridad, legibilidad, autenticidad y versatilidad en su aplicación. De esta manera, la propuesta debía ser de fácil implementación en múltiples soportes, sustratos, tamaños y plataformas, ya fuera impresa o digital, ya que el proyecto implicaba múltiples necesidades comunicativas de acuerdo a sus intereses de proyección social y comercialización. 
Caso Garittea, del campo al campus: Creación del diseño de la identidad visual de una organización a través del trabajo colaborativo entre cominidades campesinas y la academia,

En lo atinente al campo de la intervención, la tarea a desarrollar se centró en el diseño de identidad corporativa, ya que el ejercicio en una primera fase se enmarcaría principalmente en este campo, aunque después se integraron otras áreas como envase y etiquetado, diseño publicitario, diseño de interacción, diseño de producto y diseño editorial, entre otros.

Posteriormente, se analizaron las características contextuales del proyecto con el fin de establecer de manera muy precisa el escenario de acción donde se llevaría a cabo el ejercicio. Se estudiaron en detalle las particularidades de cada una de las partes involucradas en el proyecto del café, sus principales características, sus aportes y nivel de participación en esta iniciativa, aspectos tratados con amplitud en el punto "La experiencia: actores y tensiones".

Los estudiantes en este proceso de briefing realizaron un estudio detallado de referentes y proyectos similares, tanto a nivel nacional como internacional, construyendo un Benchmarking muy completo que sirvió para identificar fortalezas, debilidades y oportunidades de diseño que pudieran ser tenidas en cuenta al momento de llevar a cabo la intervención.

Uno de los aspectos fundamentales en el desarrollo del brief, fue el referido a la audiencia a la cual iría dirigida la propuesta. Este proyecto, al ser una iniciativa que se configurará y materializará a través de una cafetería en el campus universitario de la Pontificia Universidad Javeriana Cali, involucra a diversos públicos que circulan día a día por ella. Este punto pone en evidencia la diversidad de personas que deben ser tenidas en cuenta para el desarrollo del diseño de identidad para el proyecto del café, siendo éste uno de los mayores retos para la definición de requerimientos alrededor del naming y el posterior ejercicio de logotipia, así como de arquitectura de marca por parte de los estudiantes.

\section{Modelo de encuentro de trabajo colaborativo y participativo para la creación de la identidad de Garittea.}

En términos del sustento epistemológico del proyecto, destacan tres premisas fundamentales: la visión de trabajo en entornos colaborativos y principios de diseño participativo bajo un enfoque sistémico. Roncancio (2011) afirma que "un entorno colaborativo universidad - empresa es no sólo deseable sino una estrategia prometedora para promover el desarrollo humano, ambiental, económico y social para facilitar el desarrollo científico y la innovación y, especialmente, para asegurar mínimos de convivencia y cohesión social" (pág. 304). En términos de Garittea, en esta fase del proyecto se definieron los actores implicados y sus roles a través de una triada de participación, en la que cada uno de ellos aportó un valor significativo para el crecimiento y desarrollo del proyecto. La Universidad mediante el equipo de $\mathrm{I}+\mathrm{D}+\mathrm{i}$ (gestores y profesores) y los diseñadores (los estudiantes); y por su parte la organización con su knowhow y una constante comunicación en torno a saberes necesarios para la creación de la identidad. 


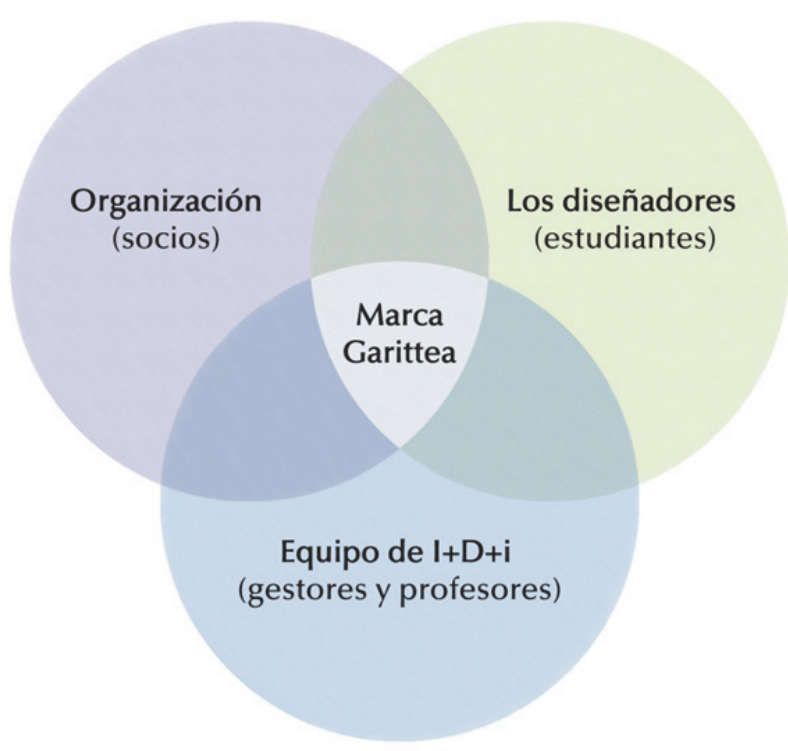

Fig. 1 Triada de trabajo colaborativo para la creación de la marca Garittea (Fuente: elaboración propia)

La perspectiva del trabajo en entornos colaborativos se basa en la propuesta de Leydesdorff (2000) y (Etzkowitz \& Leydesdorff, 2000). La primera hélice de la triada reflejada en la empresa/organización (socios de Garittea), está conformada por la asociación de pequeños Agricultores de la Marina ASOPECAM-, la Asociación de Caficultores Orgánicos de Colombia-ACOC- y el Instituto Mayor Campesino -IMCA-, los cuales han aportado el know-how y la experiencia en detalle en cuanto a sus procesos y el desarrollo de nuevos productos. Su figura como demandante del servicio en la creación de la identidad organizacional ha sido clave en la formulación del briefing y los requerimientos de diseño.

La segunda hélice estructurada por un equipo de $\mathrm{I}+\mathrm{D}+\mathrm{i}$ (profesores y gestores), todos pertenecientes a la Pontificia Universidad Javeriana Cali, los cuales y según la definición de Etzkowitz \& Leydesdorff (2000), han sido puente creador del conocimiento con un papel estratégico fundamental para generar la relación de la empresa con el diseño, articulando el conocimiento de las personas involucradas y sus relaciones, apoyando la innovación en los procesos de creación de valor, e incorporando al propio tiempo sus metodologías y herramientas bajo un enfoque multidisciplinar. Saberes como el mercadeo, la ingeniería, la economía, la psicología, la arquitectura y el diseño, han sido recogidos en modelos sistémicos de formulación por objetivos para apoyar los procesos creativos y la gestión del diseño (de la información y el conocimiento, de responsabilidades y desarrollo de las actividades en el proceso).

Por último, la tercera hélice está representada en los diseñadores (estudiantes), de varias carreras de la Pontifica Universidad Javeriana Cali, aportando insumos conceptuales clave para un resultado obtenido, incluyendo los aspectos normativos y legislativos inherentes a los productos de la marca.

En términos del diseño participativo, destacan tres cualidades esenciales señaladas por autores como Kang et. al. (2015), relacionadas con la inclusión de los actores involucrados en todas las fases del proceso, el fomento del sentido de pertenencia y el prototipado cooperativo.

Los beneficios de este ejercicio participativo y colaborativo para la universidad se ven representados en la generación de conocimiento que luego podrá ser trasmitido a generaciones siguientes de actores bajo unas estructuras dinámicas que centran el proceso en las personas. 
Caso Garittea, del campo al campus: Creación del diseño de la identidad visual de una organización a través del trabajo colaborativo entre cominidades campesinas y la academia,

\section{Modelo sistémico de formulación por objetivos como herramienta para la creación y gestión de la Identidad de la marca Garittea}

La compleja participación de los actores de Garittea en el proyecto de creación de identidad, requirió de un modelo para organizar y gestionar la información, variables y requerimientos, de tal forma que se pudiesen además evidenciar y trazar las rutas de toma de decisiones a través del proceso. Para ello se recurrió al modelo "IdThink" desarrollado por Demarchi et al. (2014), que se centra en la gestión del conocimiento inherente a los productos, servicios y procesos de las organizaciones. El "IdThink" a su vez, se asienta en el modelo sistémico de formulación por objetivos propuesto por Hernandis (1999). Se trabajaron los dos componentes marco del modelo: sistema de estudio (del modelo de producto) y sistema exterior, con sus respectivas variables de entrada y salida. El análisis del sistema exterior se llevó a cabo bajo el enfoque propuesto por Rosales, E. et al. (2015), a través de un mirador conceptual y seis componentes clave: la empresa/organización, conocer el contexto (análisis DAFO), conocer las personas, la competencia-referentes, explorando conceptos (perfil del consumidor ideal), benchmarking internoexterno y un panel semántico conceptual (moodboard). Este enfoque permite la externalización del conocimiento, y posteriormente hacerlo explícito, para favorecer el planteamiento y la formulación de los objetivos en el sistema de estudio.

Volviendo permanentemente sobre esta información, se permite el análisis, la descripción y la resolución de problemas, a los fines de realizar posteriores correcciones a desviaciones en la gestión del diseño. De igual forma, se facilitan los procesos de iteración y de validación durante las diferentes fases de prototipado cooperativo.

En el caso de Garittea, el primer paso fue determinar entre todos los actores, las claves asociadas a la creación de la identidad de una marca que permitiera mostrar la consolidación de un proyecto humano, honesto, justo, orgánico y solidario. Para los productores de café del Valle de Cauca el principal desafío que la marca debía transmitir, era el de darle valor a las riquezas que poseen en lo agroecológico y en lo cultural, además de hacer reconocimiento a la necesidad de fortalecer la cadena productiva, de manera que se pudiera llegar al consumidor final, aportando a su educación a través de la valoración del consumo de productos agroecológicos y la apreciación de la calidad del café producido bajo este modelo. 


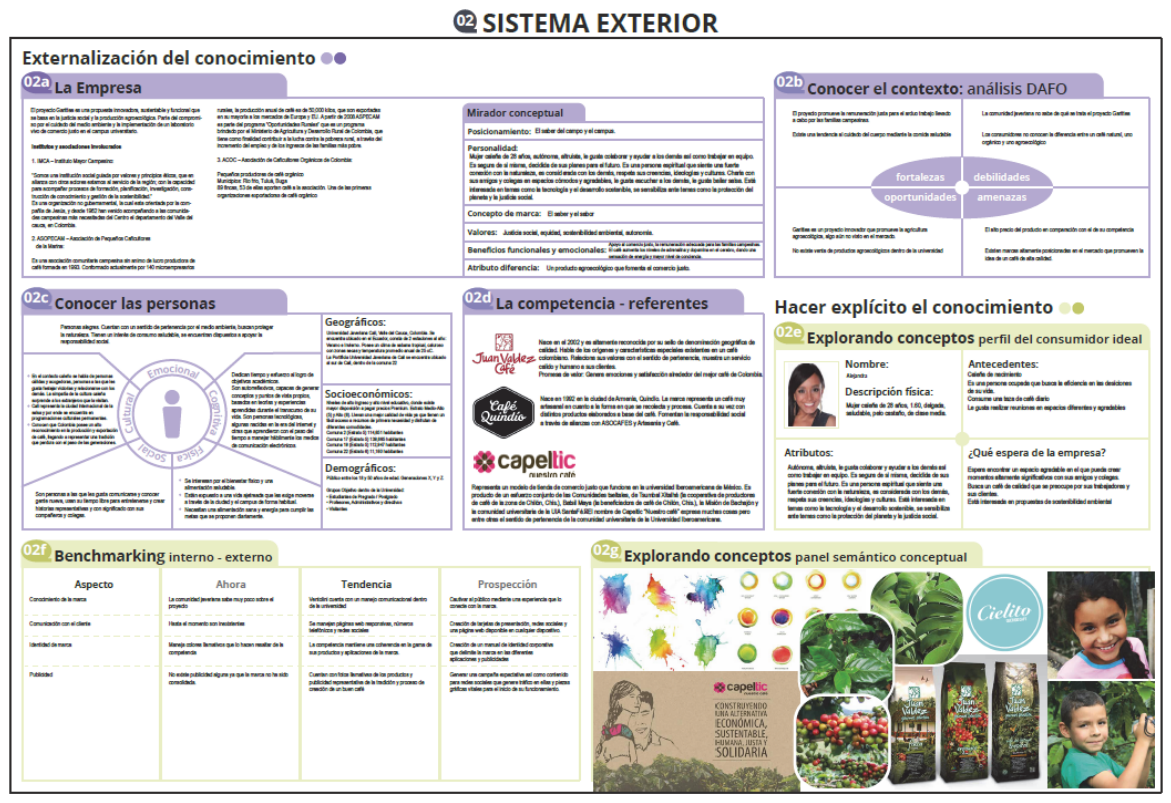

Fig 2: Análisis del sistema exterior proyecto Garittea.

En el primer componente del modelo, el sistema exterior fig 2, se recogió toda la información que afectaría al sistema de producto y en el caso de Garittea, toda la información necesaria para tomar decisiones en la creación de su identidad, los puntos fuertes que debían ser investigados así como los datos pertinentes para la empresa desde el mirador conceptual: posicionamiento, personalidad, concepto de marca, valores, beneficios funcionales y emocionales y atributos diferenciales. Se estudiaron igualmente la competencia y los referentes, incluyendo sus estrategias e identidad visual (imagen, tipografía, color y forma). Por otra parte, se estudió al usuario/consumidor desde un análisis de los factores humanos a través de las cinco dimensiones propuesta de Kumar (2013) y se analizó el contexto a través de un análisis DAFO. Por último se recurrió a un explorador de conceptos a través de un panel semántico conceptual que se emplea para descubrir asociaciones y percepciones construidas por los actores participantes del proyecto, a través de un moodboard de imágenes propias para inspirarse y dar personalidad al proceso creativo.

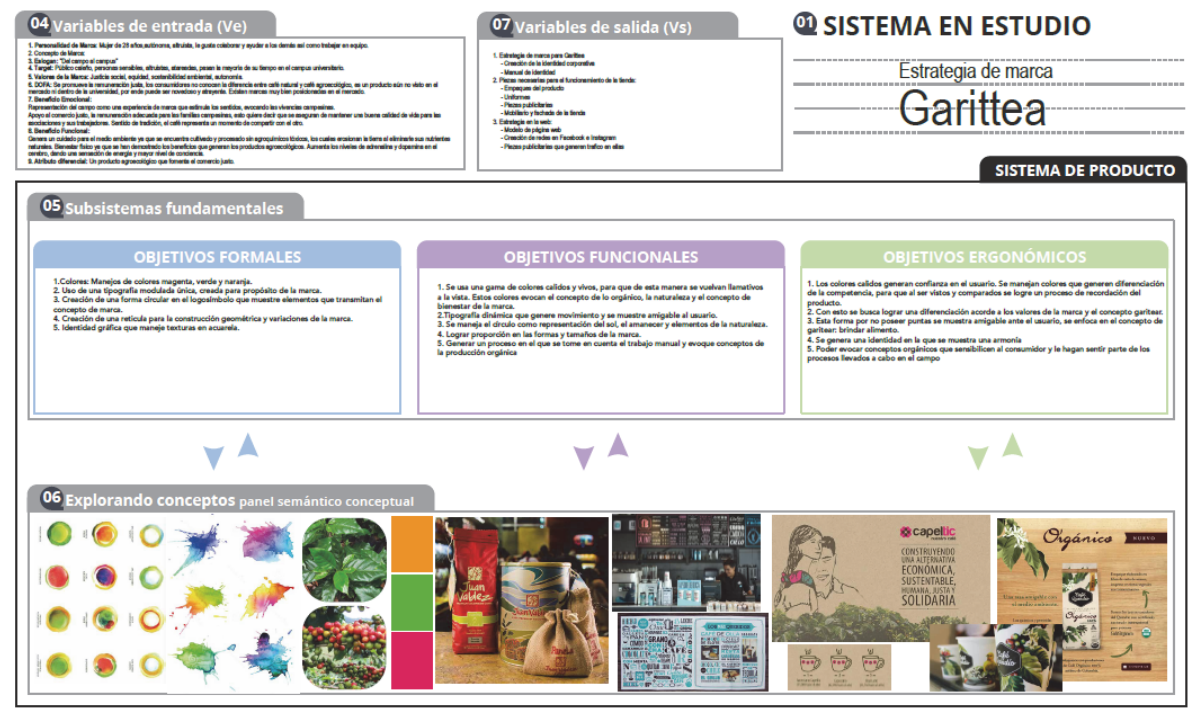

Fig 3: Análisis del sistema de producto proyecto Garittea. 
Caso Garittea, del campo al campus: Creación del diseño de la identidad visual de una organización a través del trabajo colaborativo entre cominidades campesinas y la academia,

En el segundo componente del modelo y con apoyo del ejercicio realizado en el sistema exterior, se realizó el análisis del sistema de estudio y del sistema de producto, en el que se establecieron las variables de entrada y de salida. Se volvió sobre el panel semántico conceptual y se definieron los objetivos formales, funcionales y ergonómicos de acuerdo a los criterios de Hernandis (1999) y Rosales et al. (2015). Los objetivos formales están relacionados con la satisfacción de los requerimientos estéticos de un producto (semiótica, forma, colores, texturas, tendencia, acabados, armonía, originalidad), los objetivos funcionales están asociados con proporcionar al producto la tecnología, adaptabilidad, transformación, conservación, uso, co-creación/configuración, y por su parte, los objetivos ergonómicos, se ocupan de la adecuación de los aspectos formales y funcionales al usuario (antropometría, percepción visual, aspectos cognitivos, afectivos y conductuales). Ver fig 3.

Como resultado de este proceso de creación fue una marca que refleja los valores, beneficios, misión y visión de la organización; su tipografía, simbolo visual, colores, slogan y el sistema gráfico rememora la labor diaria de las familias campesinas, el bienestar humano y la frescura de los rostros trabajadores, usando este último como elemento en el lenguaje gráfico de la marca. Para el distintivo el concepto manejado fue la agroecología evocando lo orgánico reflejado en una tipografía única e irreproducible (logotipo) y exaltando en el isotipo el propio nombre "garitear" que significa transportar alimento a la plantación para los recolectores de café. Ver fig 4.

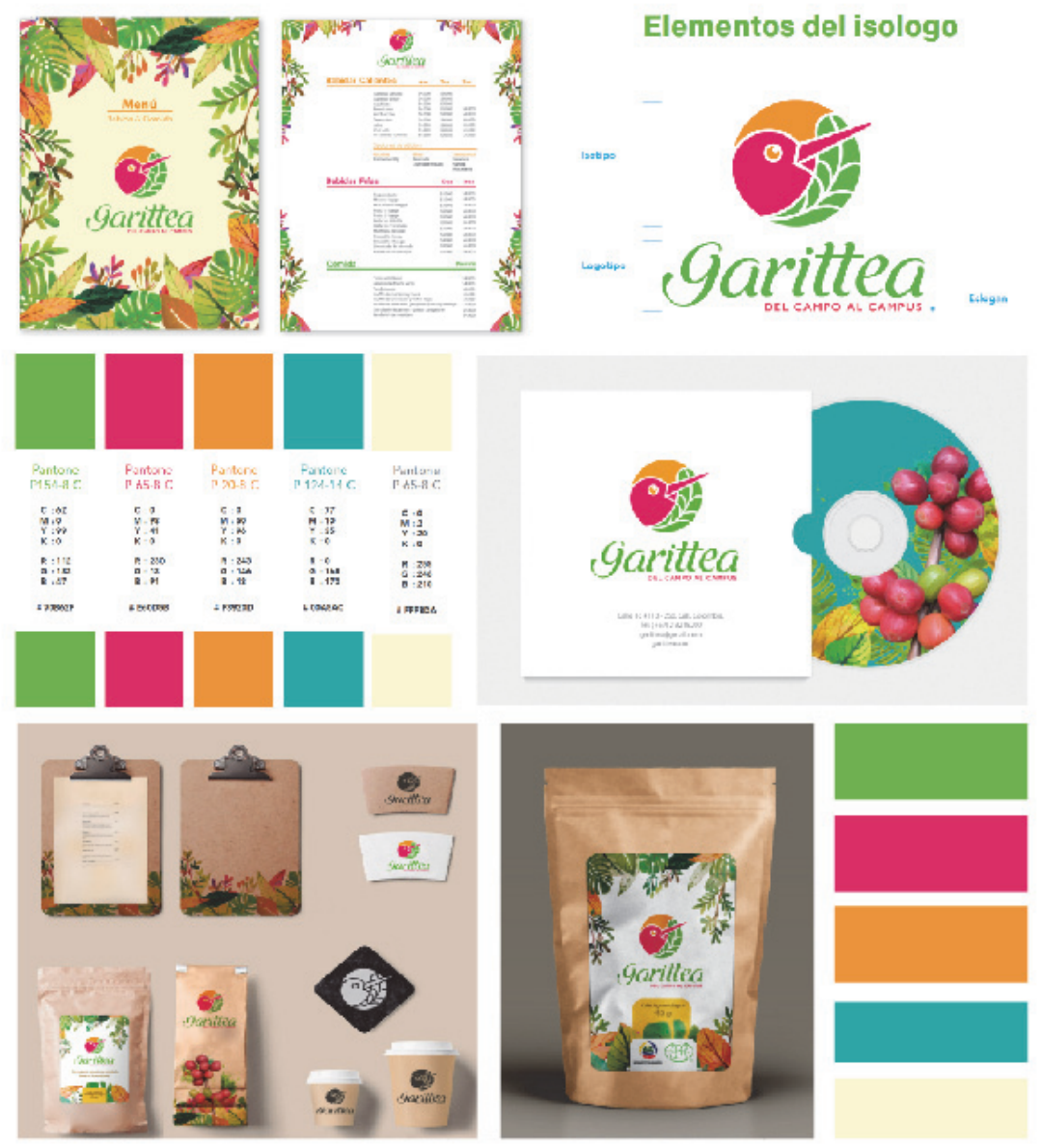

Fig 4. Diseño de la Identidad Visual de Garittea. 


\section{Algunas reflexiones para seguir abordando}

El desarrollo de un proyecto con las características que hemos enunciado, nos ubica en diferentes lugares que es necesario abordar desde la formación de diseñadores y profesionales en general. No queremos dejar pasar este ejercicio reflexivo sin compartir algunas ideas que nos han suscitado el encuentro e intercambio entre académicos, comunidades campesinas y funcionarios de una ONG.

» El acto productivo del diseño implica la creación de nuevos actores, en tanto se hace obligatoria la lectura crítica del contexto que ayude a orientar la propuesta creativa y a capturar el sentido de lo no dicho, de lo profundo, de lo fundante. Surgen algunos interrogantes: ¿Cómo lograr esta perspectiva sociocultural y política en la formación de los profesionales y superar la mediación instrumental que lleva a la materialización de la demanda del cliente?, en esa lógica globalizada del cliente tiene la razón.

» El lugar del usuario o consumidor. ¿Cómo lograr una interacción continua con el usuario para garantizar dinamismo y pertinencia en la creación de la marca? Esta y otras preguntas similares nos llevan a plantearnos la forma de cristalizar la promesa de valor del producto, seguramente la definición de la experiencia de marca exige una interacción permanente con el usuario, el consumidor final.

»Entonces, se requiere diseñar estrategias que sean flexibles, que logren capturar la identidad de la marca como una constante de sentido, pero al mismo tiempo introduzcan elementos nuevos, frescos, que den cuenta de ese mundo circular en interdependencia que obedece a lo rural. Aquí el uso del espacio, sus formas de habitarlo, hacerlo propio tiene una connotación claramente articulada a la marca, a Garittea, donde el campo, la vida silvestre llega al campus universitario, a la ciudad.

» La creación de la identidad de marca corresponde a concretar lo intangible, aquello que se fundamenta en los valores de la propuesta de negocio, de ahí que el valor del trabajo interdisciplinario y el intercambio de perspectivas y saberes se convierte en un detonador de colores, imágenes, formas y trazos, que logran dar cuenta del encuentro humano y territorial.

\section{Referencias}

ALBARRACÍN, V. (2009). El emprendimiento de la causa perdida En Giros y desvíos Una aproximación a la gestión desde las artes visuales. Ministerio de Cultura Colombia.

CHAVES, N. (2001). La Imagen Corporativa. Barcelona, España: Gustavo Gilli.

DE CERTEAU, M., GIARD, L. \& MAYOL, P. (2006). La invención de lo cotidiano 2. Unversidad Iberoamericana. México.

DEMARCHI, A., FORNASIER, C., HERNANDIS ORTUÑO, B., \& ROSALES MARQUINA, E. (2014). IDTHINK: facilitador da visualização dos conhecimentos da empresa. $11^{\circ}$ Congresso Brasileiro de Pesquisa e Desenvolvimento em Design. 1. Blucher Design Proceedings.

ETZKOWITZ, H., \& LEYDESDORFF, L. (2000). The dynamics of innovation: from National Systems and "Mode 2' to a Triple Helix of university-industry-government relations. Research Policy, 29(2), 109-123.

GARCÍA, N. (1990). Cultural Hibridas. Estrategias para entrar y salir de la modernidad. Editorial Grijalbo. México.

GIL, J. (2009). Gestión de la creación, creación de la gestión en Giros y desvios Una aproximación a la gestión desde las artes visuales. Ministerio de Cultura Colombia. 
Caso Garittea, del campo al campus: Creación del diseño de la identidad visual de una organización a través del trabajo colaborativo entre cominidades campesinas y la academia,

HERNANDIS, B., \& IRIBARREN, E. (1999). Diseño de Nuevos Productos. Una Perspectiva Sistémica. España: Centro de Formación de Postgrado. Universidad Politécnica de Valencia.

KANG, M., CHOO, P., \& WATTERS, C. E. (2015). Design for Experiencing: Participatory Design Approach with Multidisciplinary Perspectives. Procedia - Social and Behavioral Sciences, 174, 830-833.

KUMAR, V. (2013). 101 design methods. A structured approach for driving innovation in your organization. Canada: John Wiley \& sons Inc.

LEYDESDORFF, L. (febrero de 2000). The triple helix: an evolutionary model of innovations. Research Policy, 29(2), 243-245.

PHILliPS, P. L. (2004). Creating the Perfect Design Brief: How to Manage Design for Strategic Advantage. Skyhorse Publishing Inc.

RONCANCIO, P. (2011). De las capacidades dinámicas como enfoque de la estrategia a la integración de competencias para la construcción de un entorno colaborativo universidad - empresa. Revista Ciencias Estratégicas, 19(26), 295-305.

ROSAleS, E., GONZÁleZ, J., PERFETTO, A., BITTENCOURT, C., \& HERNANDIS, B. (2015). Un modelo innovador para la gestión de la identidad en las organizaciones. International Conference on Design Research Designa 2015- (págs. 235-241). Covilhã, Portugal: Labcom.IFP, Universidade da Beira Interior (UBI).

SANTOS, M. (2000). Por uma outra globalização: do pensamento único à consciência universal. Rio de Janeiro: Record, 174 p. En: Sobarzo, Oscar. Eure, Santiago. Brasil. 